\title{
A COMPARATIVE STUDY BETWEEN STANDARD MONOPOLAR VERSUS BIPOLAR SALINE TURP: OUR EXPERIENCE IN ARMED FORCES HOSPITAL.
}

\author{
MS ISLAM ${ }^{1}$, SM WAHEED ${ }^{2}$, MA RAKIB ${ }^{3}$, A CHOWDHURY $^{4}$, MN AMIN $^{5}$
}

\begin{abstract}
s
Objective: To-evaluate the outcome of bipolar Versus conventional monopolar transurethral resection of the prostate (TURP) on urinary function.

Material and Methods: A total of 300 patients with benign prostatic hyperplasia (BPH) were randomized to bipolar or monopolar conventional TURP treatment groups. Operative and early postoperative variables and complications were recorded and all patients were re-evaluated at 1, 3, 6 and 12 months after surgery using the International Prostate Symptom Score (IPSS), uroflowmetry, post-void residual urine volume (PVR).

Results: The operating time was shorter in the monopolar TURP group. Postoperative bleeding and blood transfusion requirements did not significantly differ between the two groups. Sodium levels were significantly lower in the monopolar group than in the bipolar group. Transuretheral resection syndrome developed in two (1.4\%) patients in the monopolar group. Both groups had similar and significantly improved IPSS values, maximum urinary flow rate values and PVRmeasurement.

Conclusion: Bipolar TURP is a safe and effective procedure that is associated with a relatively longer operating time, a smaller reduction in serum sodium levels and a similar efficacy compared with conventional monopolar TURP.
\end{abstract}

Key words: Transurethral resection of prostate, TURP, monopolar, bipolar

Bangladesh J. Urol. 2018; 21(2): 66-70

\section{Introduction}

The 'gold standard' surgical treatment of clinically obstructive BPH is TURP, but life-threatening complications such as transurethral resection (TUR) syndrome are occasionally observed[1]. The most important aetiological factor for TUR syndrome is the intraoperative use of hypotonic irrigation solutions such as glycine. In recent years, bipolar electrosurgical technologies with isotonic saline irrigation solutions have been implemented in an attempt to minimize BPHrelated complications [1-3]. When compared with

1. Brig. Gen. (Professor), Urology Centre, $\mathrm{CMH}$ Dhaka

2. Col. (Professor), Urology Centre, $\mathrm{CMH}$, Dhaka

3. Lt. Col.(Associate Professor), Urology Centre, $\mathrm{CMH}$, Dhaka

4. Lt. Col.(Associate Professor), Urology Centre, $\mathrm{CMH}$, Dhaka

5. Major, Urology Centre, $\mathrm{CMH}$, Dhaka

Correspondences: Islam MS, Urology Centre, $\mathrm{CMH}$, Dhaka

Received: 9 August 2017

Accepted: 05 March 2018 conventional monopolar TURP, the impact of bipolar TURP on preoperative bleeding, early postoperative outcomes (e.g blood transfusion requirements), and long-term outcomes (e.g. urethral stricture) are still debated.

We compared the preoperative, early postoperative, and long-term urinary functions of bipolar vs. conventional monopolar TURP in a prospective, randomized trial in patients with $\mathrm{BOO}$ attributable to $\mathrm{BPH}$.

\section{Material and Methods}

The study was conducted in the department of urology. Combined Military Hospital Dhaka, between February 2014 and January 2016. Three hundred men with BPHrelated LUTS were enrolled in the study and prospectively randomized into a monopolar TURP and a bipolar TURP group. Informed consent was obtained from all patients, and the study was approved by the 
institutional ethical committee. A full medical history was obtained from all patients, and the patients were evaluated preoperatively using physical examination and DRE, urine analysis, urine culture, serum electrolytes, kidney function, complete blood count, PSA, the IPSS, inclusive of the question on quality of life, and a quality-of-life (QoL) score, and uroflowmetry. In addition, prostate volumes and post-void residual urine volumes (PVRs) were measured ultrasonography.

To be included in the study, patients were required to have symptomatic BPH that required surgery owing to urinary retention or failed medical therapy. Patients with neurogenic bladder dysfunction, previous prostatic or urethral surgery, prostate cancer, bladder calculus and coagulopathy were excluded. After routine cystourethroscopy, monopolar TURP with power settings of 110 to $130 \mathrm{~W}$ and 110 to $90 \mathrm{~W}$ for cutting and coagulating currents, respectively or bipolar TURP (using an Storz Autocon II 400 ] generator with power setting of 200W for cutting and 100W for coagulation) was performed with a 26- $\mathrm{F}$ continuous flow resectoscope using mannitol or saline irrigation under general or spinal anaesthesia. At the end of each procedure, a 22-F three-way Foley catheter was inserted, and continuous irrigation saline was maintained until, urine was clear. A complete blood count and a serum electrolyte panel were evaluated in each patient during the early postoperative period. Operating time, length of hospitalization, perioperative complications, early postoperative complications, catheterization time, changes in haemoglobin levels and changes in serum electrolytes, including sodium, chloride and potassium, were recorded.

All patients were re-evaluated at 1, 3, 6 and 12 months after surgery by IPSS, QoL score, uroflowmetry and PVR.. Data were analysed using SPSS 16.0 for Windows (SPSS,USA). Statistical analysis was done by using student's t-test and chi-square test. A p-value $<0.05$ was considered as statistically significant.

\section{Results}

In the monopolar TURP group, 130 of 153 (84.9\%) patients were followed for at least 1 year, and in the bipolar TURP group, 127 of 147 (86.4\%) patients were followed for the same time period. The number of patients who dropped out of the study was similar in both groups. Of the 43 patients who dropped out, four died from myocardial infarction, one from respiratory insufficiency and one from cerebrovascular accident, and the other 39 patients were lost follow-up. The longterm outcomes of the study and their comparisons with preoperative variables were based on data from 257 patients.

Preoperative variables including mean age, IPSS, QoL, prostate volume and haemoglobin level were similar in the monopolar and bipolar groups (Table 1). In the bipolar and monopolar groups, the mean (SD) operating times were 58.7 (16.8) and $54.0(21.0)$ min, respectively $(P=0.03)$. The mean (SD) drop in postoperative haemoglobin levels after $24 \mathrm{~h}$ of follow-up was $1.2(0.9)$ $\mathrm{g} / \mathrm{dL}$ in the bipolar group and $1.41(1.23) \mathrm{g} / \mathrm{dL}$ in the monopolar group $(P=0.1)$. Blood transfusions were required in three $(2.1 \%)$ patients in the bipolar group and in eight (5.6\%) patients in the monopolar group ( $P$ $=0.2$ ).

Table-I

Preoperative demographic characteristics

\begin{tabular}{lccc}
\hline $\begin{array}{l}\text { Preoperative } \\
\text { variables* }\end{array}$ & $\begin{array}{c}\text { Monopolar } \\
\text { group }\end{array}$ & $\begin{array}{c}\text { Bipolar } \\
\text { group }\end{array}$ & $\begin{array}{c}\text { P } \\
\text { Value }\end{array}$ \\
\hline Age, years & $67.7(7.2)$ & $67.4(9.2)$ & 0.76 \\
IPSS score & $18.5(2.4)$ & $18.6(2.3)$ & 0.32 \\
Qol score & $4.0(0.7)$ & $4.1(0.8)$ & 0.24 \\
Qmax ml/s & $8.0(3.5)$ & $7.3(2.8)$ & 0.40 \\
PVR, ml & $106.9(61.6)$ & $117.8(77.6)$ & 0.33 \\
Prostate & $55.9(22.8)$ & $57.9(24.8)$ & 0.76 \\
volume, ml & & & \\
\hline
\end{tabular}

*Values are reported as mean (SD).

The changes in the electrolyte concentrations were similar in both groups, with the exception of sodium (Table-II). The mean postoperative sodium level was relatively lower in the monopolar group than in the bipolar group. TUR syndrome developed in two (1.4\%) patients who underwent monopolar TURP, but no patients in the bipolar group developed this syndrome. Patients in the bipolar group were catheterized for a mean (SD; range) of $2.4(1.0 ; 1-5)$ days, and the mean (SD; range) catheterization time in the TURP group was $2.6(1.2 ; 1-7)$ days. There was no significant difference in the length of hospital stays between the two groups. Monopolar TURP patients were discharged home at a mean (SD; range) $2.7(1.4 ; 1-16)$ days, and 
Table-II

2 comparative evaluation of the change in electrolyte and haemoglobin levels.

\begin{tabular}{lccc}
\hline Variable & Monopolar group & Bipolar group & P Value \\
\hline Na, mEq/L & & & \\
Preoperative & $140.3(2.7)$ & $140.5(2.6)$ & 0.70 \\
Postoperative & $137.6(5.6)$ & $139.1(3.3)$ & 0.004 \\
Mean change & $-2.82(5.8)$ & $-1.30(3.8)$ & 0.03 \\
K, mEq/L & & & 0.65 \\
Preoperative & $4.3(0.4)$ & $3.8(0.5)$ & 0.91 \\
Postoperative & $4.1(0.4)$ & $4.1(0.2)$ & 0.06 \\
Mean change & $-0.18(0.4)$ & $0.03(0.8)$ & \\
Cl, mEq/L & & & 0.08 \\
Preoperative & $103.5(4.0)$ & $104.2(3.6)$ & 0.01 \\
Postoperative & $102.2(5.5)$ & $104.4(6.1)$ & 0.16 \\
Mean change & $-1.27(6.0)$ & $0.16(5.9)$ & 0.62 \\
Haemoglobin g/dl & & & 0.72 \\
Preoperative & 13.2 & $13.1(1.3)$ & 0.09 \\
Postoperative & 11.8 & $11.9(1.4)$ & $1.2(0.9)$ \\
Mean change & 1.41 & &
\end{tabular}

Values are reported as mean (SD) SD standard deviation

bipolar TURP patients were discharged at a mean (SD; range) of $2.5(1.3 ; 1-13)$ days. Five patients in the bipolar and six patients in the monopolar group complained of some degree of incontinence, and all of these patients spontaneously recovered within 6 months.

Re-catheterization was needed in two patients because of clot retention in the monopolar group and one patient in the bipolar group.

The mean (SD) preoperative IPSS score decreased to 10.3 (3.0) and 10.8 (2.9) in the bipolar and monopolar groups, respectively (Fig. 1). The mean (SD) maximum urinary flow rate (Qmax) values increased over the 12month period from 7.2 (3.1) to 17.1 (3.1) in the bipolar group and from 8.0 (3.6) to 16.3 (4.7) in the monopolar group $(P<0.001$ for both groups; (Fig. 2). The improvements in Qmax and IPSS were similar in the two groups (Figs 1,2). In preoperatively catheterized patients, the mean (SD) Qmax values and IPSS scores over the 12-month follow-up from 14.5 (3.8) to 9.5 (2.5) in the monopolar group; 16.4 (4.8) and 8.6 (1.9) in the bipolar group, respectively. PVRs decreased significantly in regularly followed patients (Fig. 3). Maximum improvements in the Qmax values and IPSS scores were achieved at 3 and 6 months, respectively, but decreases in PVR persisted throughout the 12month follow-up periods.

After 1 year of follow-up, re-operations or dilatations owing to urethral stricture or bladder-neck contracture were required in eight patients $(6.3 \%)$ patients in the bipolar group and in six patients $(4.6 \%)$ in the monopolar group $(P=0.7)$.

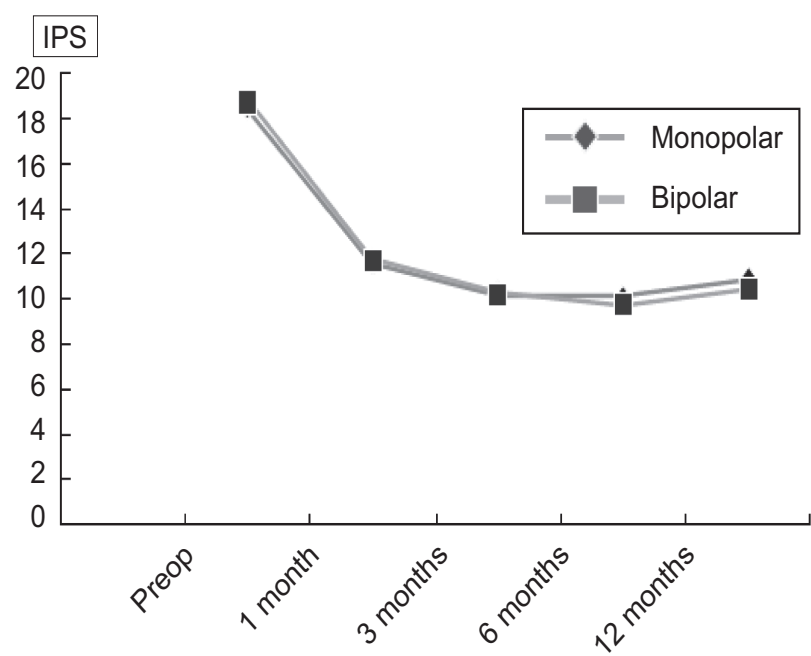

Fig.-1: Comparative evaluation of the effect of both monopolar and bipolar TURP on IPSS 
A Comparative Study between Standard Monopolar versus Bipolar Saline TURP: Our Experience in Armed Forces Hospital.

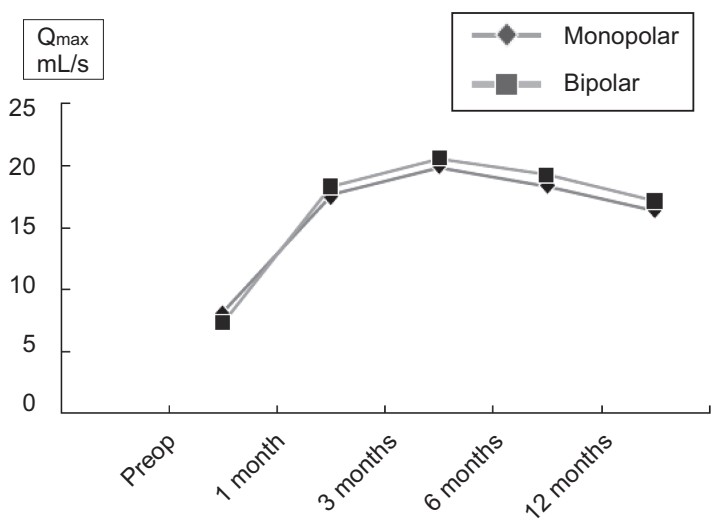

Fig.2 Comparative evaluation of the effects of both monopolar and bipolar TURP on $Q_{\max }$

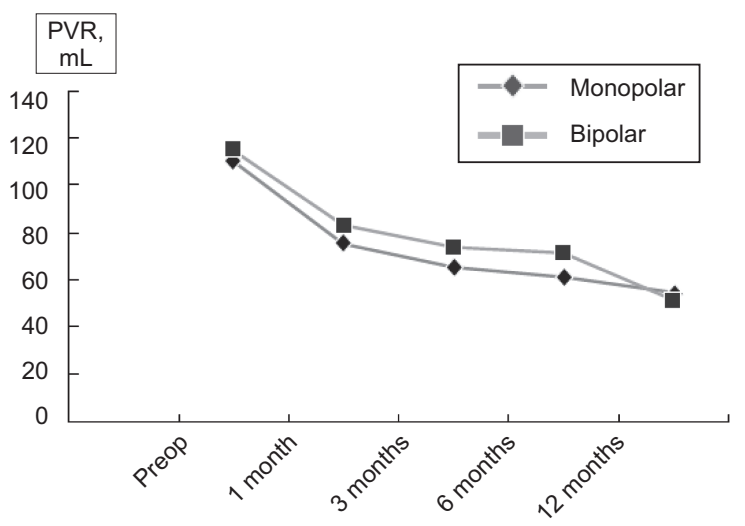

Fig.3 Comparison of time related changes in PVR in both monopolar and bipolar TURP groups.

\section{Discussion}

Bipolar TURP is performed with a saline irrigating fluid instead of glycine. Using saline during the resection protects against TUR syndrome, which is one of the important potential complications of TURP. In addition, bipolar TURP promotes better haemostasis and decreases overall complications when compared with standard monopolar TURP 5 . In the present study, operating times were little longer in the bipolar TURP group in the early part 07 surgery than in the monopolargroup. Some studies have reported longer operating times for bipolar TURPs, whereas others have reported that operating times are similar between the two groups [2,5,6].

Various studies have reported that the amount of perioperative bleeding is greater in the monopolar groups than in the bipolar groups [5]. Fagerström et al. [5] reported that the mean blood loss in the bipolar group was $235 \mathrm{~mL}$ compared with $350 \mathrm{~mL}$ in the monopolar group ( $P=0.001)$. In their study, blood transfusions were needed in $4 \%$ of the patients in the bipolar group and $11 \%$ of patients in the monopolar group $(P<0.01)$. In the present study, the decrease in the mean concentration of haemoglobin in the monopolar group was greater than that of the bipolar group, but the difference was not significant. During the early postoperative period, blood transfusions were required in three $(2.1 \%)$ and eight $(5.6 \%)$ patients in the bipolar and monopolar groups, respectively. Similarly, Ho et al. [1] observed larger decreases in the haemoglobin concentrations in their monopolar group with no significant difference $(1.2 \mathrm{mg} / \mathrm{dL}$ in the bipolar group and $1.8 \mathrm{mg} / \mathrm{dL}$ in the monopolar group). This small difference between the haemoglobin values could be attributable to two factors. First, during bipolar TURP, small venous bleedings that minimally affect the haemoglobin levels are cauterized. Second, during monopolar resection, experienced surgeons do not pay attention to small venous bleedings and thus do not cauterize them.

One of the most potentially serious complications of TURP is TUR syndrome. The development of TUR syndrome is closely related to capsule perforation and increased fluid absorption during prolonged operations. Chen et al. [4] carried out a randomized clinical study, with 2-year follow-up, comparing bipolar with monopolar TURPs. They reported decreases in mean postoperative serum sodium levels for the bipolar and monopolar TURP groups of 3.2 and $10.7 \mathrm{mmol} / \mathrm{L}$, respectively $(P<0.01)$ [4]. In the monopolar arm of the randomized study by Ho et al. [1], symptomatic TUR syndrome was detected. The operating times for their two patients were $>70 \mathrm{~min}$. In the present study, a significant decrease was detected in the mean sodium concentration of the monopolar group when compared to that of the bipolar group. TUR syndrome developed in two patients in the monopolar group, and these patients were followed up in the intensive care unit. By contrast, none of the patients in the bipolar TURPgroup developed TUR syndrome.

In the present study, the hospital stays and catheterization times were similar in the bipolar and monopolar groups. Seckiner et al. [7] prospectively compared bipolar and monopolar TURPs in a 1:1 randomization study and reported similar hospital stays and catheterization times.Indeed, hospital stay can occasionally be shorter in the bipolar group owing to a 
decreased requirement for post-TURP irrigation. In our clinical practice, however, the patient is discharged from hospital if he has not developed fever or significant haematuria after the removal of the urinary catheter and if he is able to urinate spontaneously. Nevertheless, similar studies have reported that a significant decrease in catheterization and length of hospital stay could be achieved using bipolar energy sources. In one of these studies, lori et al. [8] randomized 120 patients with LUTS into a Gyrus PlasmaKineticTM system or standard TURP and found significantly shorter catheterization times in the plasmakinetic group.

The present results confirm that both the bipolar and monopolar techniques of performing TURP reduce IPSS scores and PVR and improve urinary flow. The most important reason for this improvement is the complete removal of the obstructive prostatic tissue. According to the present results, maximum improvements are observed at 3 postoperative months for Qmax values and at 6 months for IPSS scores; however, PVR consistently decreases over 12 months.

Debates are ongoing concerning the impact of bipolar and monopolar techniques on the formation of urethral strictures. Some authors have reported higher rates of urethral strictures related to bipolar TURPs [9], whereas others have reported similar rates between the two techniques $[2,4,10]$. In the present study, the number of urethral strictures requiring surgery or dilatation was not significantly different between the groups. This result was similar to the results of the multicentre study by Mamoulakis et al. [10], who observed the development of urethral strictures in $1.4 \%$ of patients treated with bipolar TURP and in $3.6 \%$ patients treated with monopolar TURP. Urge incontinence was observed in $4.6 \%$ and $3.9 \%$ of the patients in the monopolar and bipolar groups, respectively. The symptoms of all patients with urge incontinence were improved within the first 6 months, which may be related to application of higher energy on the prostate capsule.

\section{Conclusion}

Bipolar TURP is a safe and effective procedure that is associated with relatively longer operating times, at the signing but almost similar in course of team lower reductions in serum sodium levels and similar improvements in urinary symptoms during 12 months of follow-up when compared with conventional monopolar TURP.

\section{References}

1 Ho HS, Yip SK, Lim KB, Fook S, Foo KT, Cheng $\mathrm{CW}$. A prospective randomized study comparing monopolar and bipolar transurethral resection of prostate using transurethral resection in saline (TURIS) system. Eur Urol 2007; 52: 517-22

2 Michielsen DP, Debacker T, De Boe V et al. Bipolar transurethral resection in saline-an alternative surgical treatment for bladder outlet obstruction? J Urol 2007; 178: 2035-9

3 Starkman JS, Santucci RA. Comparison of bipolar transurethral resection of the prostate with standard transurethral prostatectomy: shorter stay, earlier catheter removal and fewer complications. BJU Int 2005; 95: 69-71.

4 Chen Q, Zhang L, Fan QL, Zhou J, Peng YB, Wang Z. Bipolar transurethral resection in saline vs traditional monopolar resection of the prostate: results of a randomized trial with a 2-year followup. BJU Int 2010; 106: 1339-43.

5 Fagerström T, Nyman CR, Hahn RG. Bipolar transurethral resection of the prostate causes less bleeding than the monopolar technique: a singlecentre randomized trial of 202 patients. BJU Int 2010; 105: 1560-4.

6 Fagerström T, Nyman CR, Hahn RG. Complications and clinical outcome 18 months after bipolar and monopolar transurethral resection of the prostate. $J$ Endourol 2011; 25: 1043-9.

7 Seckiner I, Yesilli C, Akduman B et al. A prospective randomized study for comparing bipolar plasmakinetic resection of the prostate with standard TURP. Urol Int 2006; 76: 139-43.

8 Iori F, Franco G, Leonardo C et al. Bipolar transurethral resection of prostate: clinical and urodynamic evaluation. Urology 2008; 71: 252-5.

9 Tefekli A, Muslumanoglu AY, Baykal M, Binbay M, Tas A, Altunrende F. A hybrid technique using bipolar energy in transurethral prostate surgery: a prospective, randomized comparison. J Urol 2005; 174: 1339-43.

10 Mamoulakis C, Skolarikos A, Schulze M et al. Results from an international multicentre doubleblind randomized controlled trial on the perioperative efficacy and safety of bipolar vs monopolar transurethral resection of the prostate. BJU Int 2012; 109: 240-8. 